\title{
Fetal and neonatal outcomes in intrahepatic cholestasis of pregnancy
}

\author{
Parul Sharma*, Bani Sarkar, Bangali Majhi
}

Department of Obstetrics and Gynecology, PGIMER and Dr. Ram Manohar Lohia Hospital, New Delhi, Delhi, India

Received: 20 July 2018

Accepted: 14 September 2018

\author{
*Correspondence: \\ Dr. Parul Sharma, \\ E-mail: 1990sharma.parul@gmail.com
}

Copyright: () the author(s), publisher and licensee Medip Academy. This is an open-access article distributed under the terms of the Creative Commons Attribution Non-Commercial License, which permits unrestricted non-commercial use, distribution, and reproduction in any medium, provided the original work is properly cited.

\begin{abstract}
Background: Intrahepatic cholestasis of pregnancy has been associated with adverse fetal and neonatal outcomes. The objective of this study was to assess the improvement in fetal and neonatal outcomes with timely intervention in pregnant females with intrahepatic cholestasis of pregnancy

Methods: This prospective observational study included 50 patients attending antenatal clinic of the Department of Obstetrics and Gynecology of Dr. RML Hospital, from 1st November 2015 to 31st March 2017, using inclusion and exclusion criteria. History, examination, blood investigations, with ultrasound upper abdomen was done for all the patients. Diagnosed patients were treated accordingly and fetal and neonatal outcomes were studied. The data was analyzed using STATA (version 13.1, Stata Corp 4905 Lakeway Drive College Station, Texas 77845 USA). Comparisons were analyzed using Chi-Square, Student's t-tests.

Results: In the present study, in view of timely diagnosis and intervention, $82 \%$ of the neonates had birth weight $>2.5 \mathrm{~kg}$. $94 \%$ of the total neonates had $>7$ APGAR at $1 \mathrm{~min}$, and $100 \%$ of the neonates had $>7$ APGAR at $5 \mathrm{~min}$. No stillbirth was observed in present study group. No NICU admission was observed in the present study.

Conclusions: Patients with intrahepatic cholestasis of pregnancy should be taken with utmost care. With early intervention, the adverse effects on fetus and neonates can be reduced to a significant amount.
\end{abstract}

Keywords: APGAR, Fetal, Intrahepatic cholestasis, NICU, neonatal

\section{INTRODUCTION}

Intrahepatic cholestasis of pregnancy is a common liver disease during pregnancy with reported incidence rates between 0.4 and $15 \%$ in different countries and populations. ${ }^{1-3}$ Intrahepatic cholestasis of pregnancy was originally described in 1883 by Ahlfeld as recurrent jaundice in pregnancy that resolved following delivery.

It predominantly occurs during the third trimester of pregnancy and subsides after delivery and is characterized by pruritus without a rash which may affect all areas of the body but characteristically starts in the soles of the feet and palms of the hands and progresses to the trunk and face, and also causes biochemical disturbances in liver tests. Intrahepatic cholestasis of pregnancy poses little maternal risk, but it has been found to be associated with increased risks of preterm delivery, meconium staining of amniotic fluid, fetal bradycardia, asphyxial events, fetal distress, and fetal demise. . $^{3,4}$

Intrahepatic cholestasis of pregnancy carries a substantial recurrence risk and has a tendency toward familial clustering. ${ }^{2,5}$

Varying findings may also be attributed to changes in the management of Intrahepatic cholestasis of pregnancy in the last two decades, by administration of ursodeoxycholic acid (UDCA) for relief from pruritus and biochemically reduction of maternal bile acids and 
induction of delivery in gestational weeks 37-38, aiming to avoid stillbirth. ${ }^{3,6-8}$

This study is done to evaluate the improvement in fetal and neonatal outcomes in such pregnancies in an Indian population receiving modern obstetric care.

\section{METHODS}

This prospective observational study was conducted at the Department of Obstetrics and Gynaecology, Postgraduate Institute of Medical Education and Research (PGIMER), Dr R.M.L Hospital, New Delhi over a period of 1 year and 4 months from November 2015 to March 2017. This study was approved by the institutional ethics and research review board. Fifty patients were enrolled randomly from amongst the patients attending antenatal clinic at PGIMER, Dr. Ram Manohar Lohia Hospital who fulfilled the inclusion and exclusion criteria.

\section{Inclusion criteria}

- Primigravidae or multigravidae women attending antenatal clinic, after 30weeks of gestation, booked at Dr. Ram Manohar Lohia Hospital, New Delhi with pruritis without a rash and with itching on the palms and soles of the feet, worsening at night.

- History of IHCP in previous pregnancy.

\section{Exclusion criteria}

- Pregnant females with known liver disease.

- Pregnant females with pregnancy induced hypertension.

- Pregnant females with known skin condition.

Detailed history followed by general physical and systemic examination was done. At the time of enrolment in the study following investigations were done: Liver function tests, serum bile acid levels, viral markers, coagulation profile, Ultrasound upper abdomen. $20 \mathrm{ml}$ fasting blood sample was collected from each patient by venipuncture in plain vial, which was centrifuged at 2800-3200 revolutions per minute for $10-15 \mathrm{~min}$ to obtain clear serum for performing LFTs and bile acids. LFT's were measured by Dry chemistry technology using Vitros-350 and bile acids by using enzyme cycling method (diazyme).

Ursodeoxycholic acid was given to improve pruritis and liver function, dose of which was adjusted according to the derangement. Repeat LFTs were done weekly and dosage modified accordingly. Termination of pregnancy was offered at 37 weeks of pregnancy or earlier in cases of fetal distress.

The fetal and neonatal outcomes were analysed in terms of stillbirth, mode of delivery, birth weight, neonatal unit admission, meconium stained amniotic fluid, and APGAR at 1 and 5 min.

\section{RESULTS}

In the study, total number of patients were 50, out of which majority were between the age group of 25-30 years, constituting around $56 \%$ of the total study group, with around $58 \%$ primigravida patients (Figure 1).

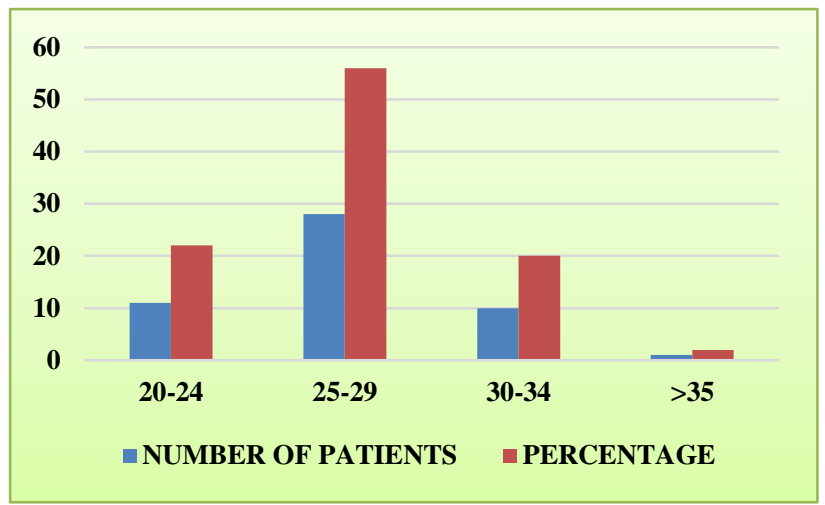

Figure 1: Age distribution of the study group.

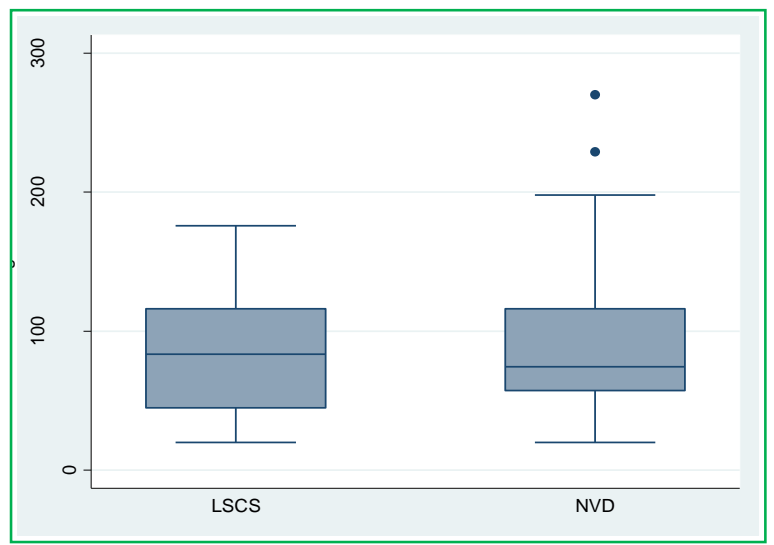

Figure 2: Association of liver function tests and bile acids with the neonatal outcome-association of SGOT with the mode of delivery.

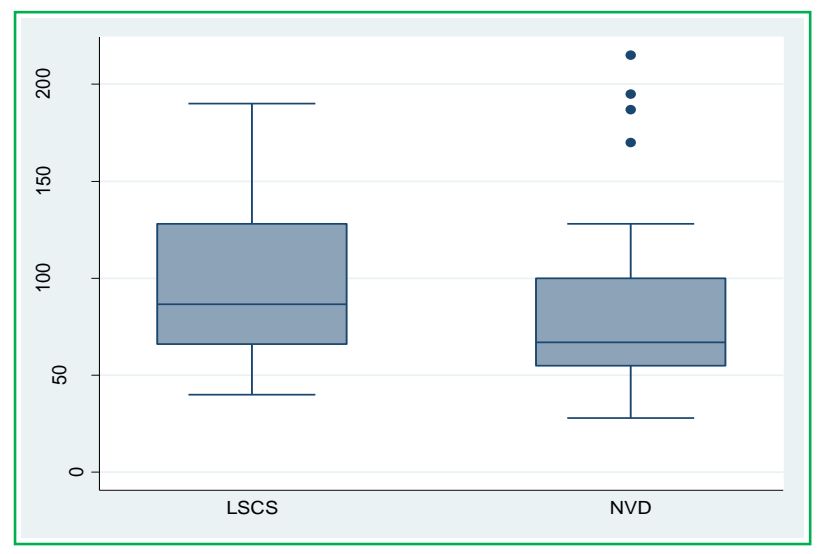

Figure 3: Association of liver function tests and bile acids with the neonatal outcome-association of SGPT with the mode of delivery. 


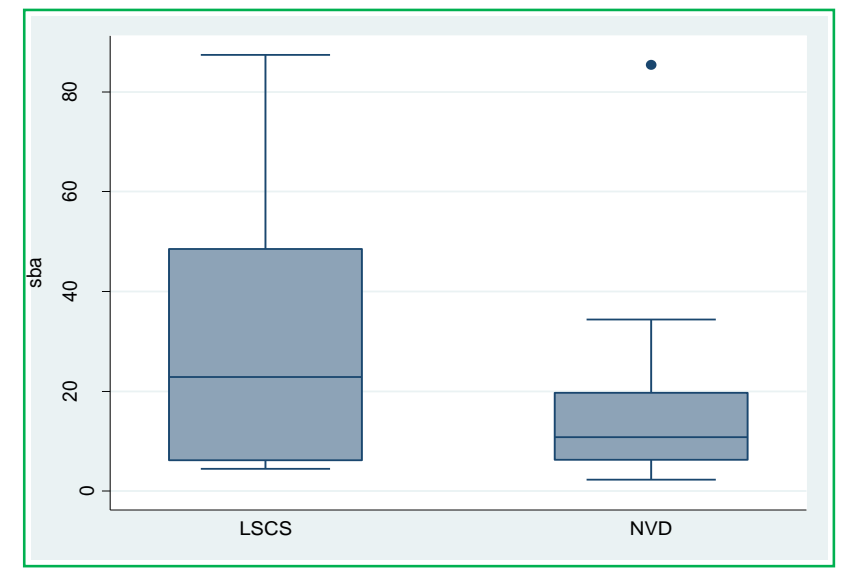

Figure 4: Association of liver function tests and bile acids with the neonatal outcome-association of SBA with the mode of delivery.

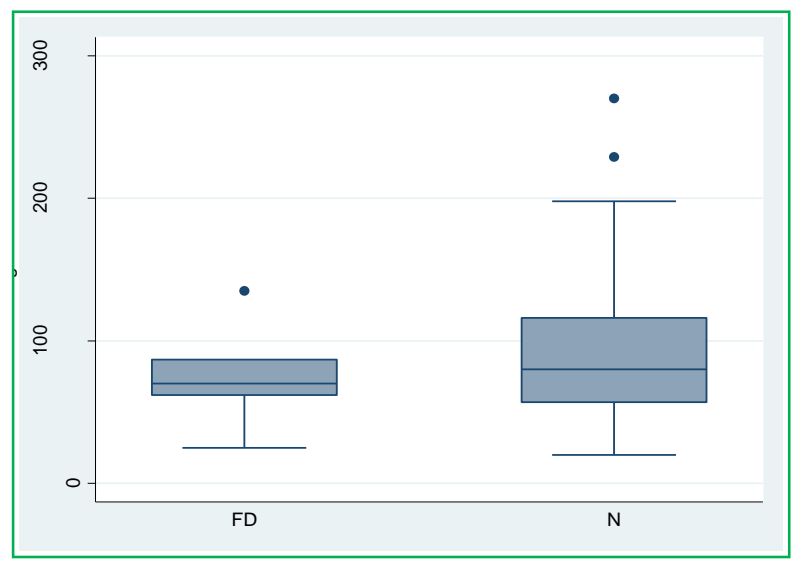

Figure 5: Association of SGOT with neonatal outcome.

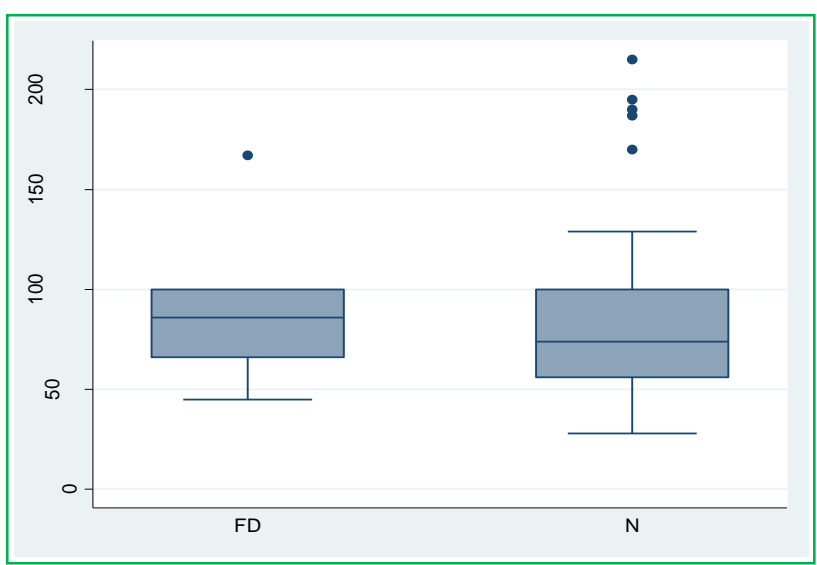

Figure 6: Association of SGPT with neonatal outcome.

The median level of the liver function test parameter and SBA with its respective range is reported in Table 1, Figure 2-4 and Table 2, Figure 5-7 and compared with the mode of delivery and neonatal outcome for any statistical significance respectively.

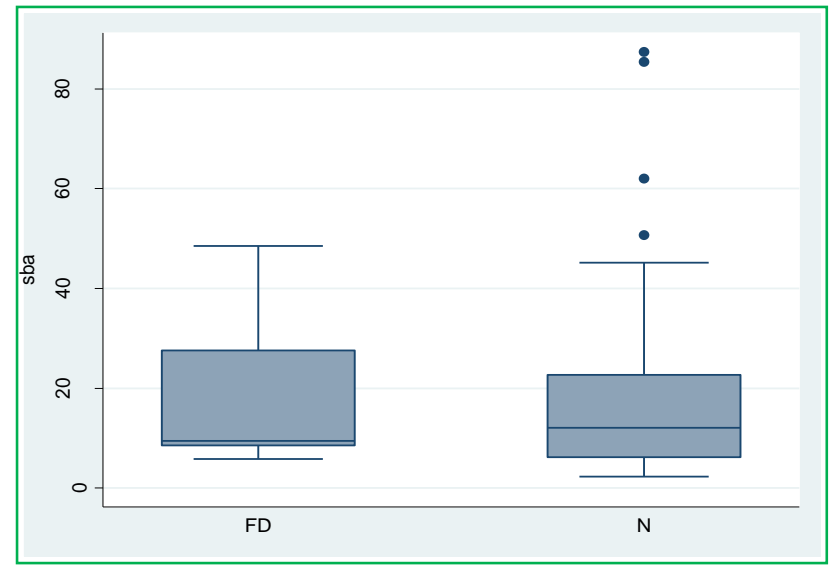

Figure 7: Association of SBA with neonatal outcome.

SGOT and SGPT were not significantly associated with the type of delivery but a marginal association of SBA with the type of delivery with $0.10 \mathrm{p}$ value indicating that the association was clinically meaningful.

Table 1: Association of mode of delivery with liver function test parameter and SBA.

\begin{tabular}{|llll}
$\begin{array}{l}\text { Clinical } \\
\text { parameters }\end{array}$ & $\begin{array}{l}\text { LSCS } \\
\text { median } \\
(\text { min-max })\end{array}$ & $\begin{array}{l}\text { NVD } \\
\text { median } \\
\text { (min-max) }\end{array}$ & p-value \\
\hline SGOT & $83.5(20.0-176)$ & $74.5(20-270)$ & 0.905 \\
\hline SGPT & $86.5(40-190)$ & $67(28-215)$ & 0.331 \\
\hline SBA & $22.8(4.4-87.4)$ & $10.8(2.3-85.4)$ & 0.101 \\
\hline
\end{tabular}

As reported in the Table 2, all the three parameters were not significantly associated with the neonatal outcome.

Table 2: Association of neonatal outcome with liver function test parameter and SBA.

\begin{tabular}{|llll|}
$\begin{array}{l}\text { Clinical } \\
\text { parameters }\end{array}$ & $\begin{array}{l}\text { Fetal distress } \\
\text { (FD) median } \\
\text { (min-max) }\end{array}$ & $\begin{array}{l}\text { Normal }(\mathbf{N}) \\
\text { median } \\
\text { (min-max) }\end{array}$ & p value \\
\hline SGOT & $70(25-135)$ & $80(20-270)$ & 0.905 \\
\hline SGPT & $86(45-167)$ & $74(28-215)$ & 0.759 \\
\hline SBA & $9.5(5.8-48.5)$ & $12.1(2.3-87.4)$ & 0.834 \\
\hline
\end{tabular}

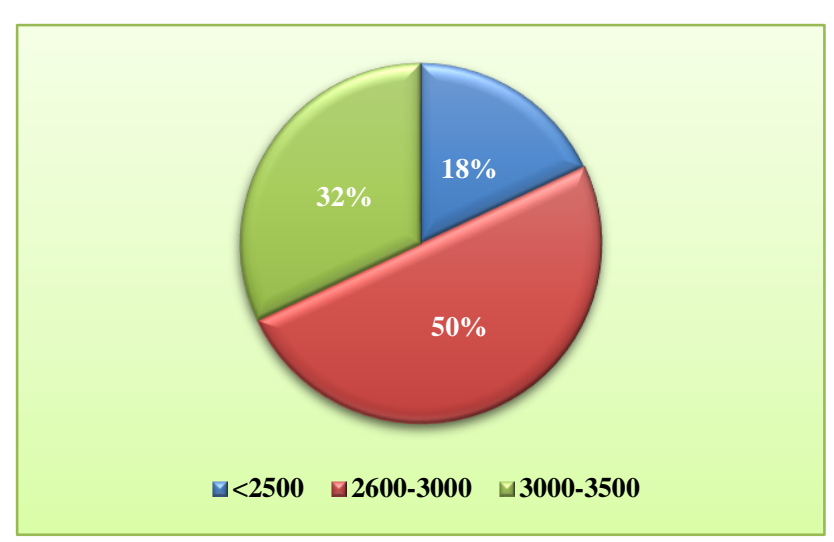

Figure 8: Birth weight distribution (gm). 
Table 3: APGAR at 1 min.

\begin{tabular}{|lll|}
\hline APGAR & 1 min & Percentage \\
\hline$<7$ & 3 & 6 \\
\hline$>7$ & 47 & 94 \\
\hline
\end{tabular}

In this study, better neonatal outcomes were seen with only $18 \%$ of the neonates falling into low birth weight criteria (Figure 8), around $94 \%$ of the total neonates having $>7$ APGAR at $1 \mathrm{~min}$, and $100 \%$ of the neonates having $>7$ APGAR at $5 \mathrm{~min}$ (Table 3 and Table 4). No stillbirth was observed in present study group. No NICU admission was observed in the present study.

Table 4: APGAR at 5 min.

\begin{tabular}{|lll|}
\hline APGAR & 5 min & Percentage \\
\hline$<7$ & 0 & 0 \\
\hline$>7$ & 100 & 100 \\
\hline
\end{tabular}

\section{DISCUSSION}

Intrahepatic cholestasis of pregnancy is a reversible cholestasis of pregnancy, occurring in late second and third trimester, which complicates maternal as well as fetal outcome, with overall incidence being 1.2-1.5\% of pregnant Indian women. Therefore, it needs to be diagnosed at an early stage to prevent complications.

It is a diagnosis of exclusion and includes both physical examination as well as certain pre-defined lab parameters. In IHCP, most important liver functions which are considered for the diagnosis are serum transaminases (SGOT, SGPT). The most characteristic lab abnormality is high serum bile acid levels (10micromol/1), on the basis of which severity of IHCP is determined. IHCP is considered severe and the risk to the fetus increases significantly when SBA levels exceed 40micromol/1. ${ }^{9}$

Management involves relief of pruritis and reducing the perinatal risks and safe delivery, for which local application of calamine lotion plus UDCA is given. Ursodeoxycholic acid is so far the most effective treatment for IHCP. Inj Vit $\mathrm{K}$ is also given to reduce the risk of $\mathrm{PPH}$ and fetal or neonatal bleeding.

Till date there is no specific test for fetal monitoring to prevent stillbirth in these patients. Therefore, continuous fetal monitoring in labour is done. As for the delivery, most obstetricians plan an elective delivery at 37-38 weeks to avoid adverse consequences.

In the present scenario, gold standard for diagnosing IHCP is Serum Bile Acids. The tests for detecting SBA are available only in selected centres and that too is costly, making liver function tests, a more accessible test for diagnosing IHCP, in accordance with the studies of Davis et al and Palma et al. ${ }^{10,11}$
In the present study, 50 pregnant females, attending the antenatal clinic, satisfying the inclusion and the exclusion criteria underwent a set of lab investigations and accordingly were diagnosed to be having Intrahepatic cholestasis of pregnancy. The study population included pregnant females with no pre-defined age group. Mostly patients fell into age group between 24-28years. In the present study, study population included both primi and multigravida patients. Primi patients were $58 \%$ of the total study group. There was no previous history of IHCP in patients having second or third pregnancy, and there was no family history found among the study group. Rook et al concluded the same in his study. ${ }^{12}$

These patients were started on ursodeoxycholic acid, dosage of which was adjusted according to the weekly LFT's and SBA. There was significant improvement symptomatically and in lab parameters with timely and judicious usage of ursodeoxycholic acid, and the fetal and neonatal outcomes were also better. This was in accordance with the studies of Bacq et al, Benjaminov et al, Kondrackiene et al and Glantz et al. ${ }^{6,13-15}$

Zhonghua et al conducted a prospective cohort study to evaluate the relationship between IHCP and neonatal outcomes and observed that IHCP was associated with low birth weight babies and adverse neonatal outcomes. ${ }^{16}$

In the present study, in view of timely diagnosis and intervention, only $18 \%$ of the neonates had low birth weight, which in itself suggests improved neonatal outcome on starting the treatment on time.

Turkmen GG et al conducted a retrospective comparative study in which he divided study group into cases and controls, on the basis of IHCP, and observed that the mean birth weight of the control group was statistically significantly higher than in the case group. ${ }^{17}$

Garcia-Flores $\mathbf{J}$ et al, conducted a prospective observational study in 145 pregnant patients with persistent pruritis, and concluded that IHCP patients had higher rate of NICU admission and overall neonatal morbidity. ${ }^{18}$

Brouwers et al study concluded the same. ${ }^{19}$ The mode of delivery and the neonatal outcomes significantly improved in present study group due to timely diagnosis and treatment of patients and therefore, no significant statistical difference was observed between liver function parameters and SBA with the mode of delivery or neonatal outcome, as shown in Table 1 and 2. (Figure 27). No stillbirth and no NICU admission were observed in present study group.

Shemer EW et al conducted a population-based cohort study, in which he concluded that patients with IHCP were more likely to have a neonate with $<7$ APGAR score at $5 \mathrm{~min}^{20}$ 
In the present study, APGAR at $1 \mathrm{~min}$ and APGAR at $5 \mathrm{~min}$ were observed as a marker for neonatal outcome, and it was observed that only $6 \%$ of neonates had APGAR $<7$ at $1 \mathrm{~min}$, and none of the neonate had APGAR $<7$ at $5 \mathrm{~min}$, as shown in Table 3 and 4 , thus emphasizing the timely intervention in cases of IHCP.

Sharma $\mathrm{N}$ et al conducted an observational study in which diagnosed cases of IHCP were given medical and active management and observed lesser incidence of low (<7) APGAR score. ${ }^{21}$

\section{CONCLUSION}

The study concluded that patients with IHCP should be taken with utmost care and for the good fetal and neonatal outcome, timely and early intervention should be done.

Funding: No funding sources

Conflict of interest: None declared

Ethical approval: The study was approved by the Institutional Ethics Committee

\section{REFERENCES}

1. Joshi D, James A, Quaglia A, Westbrook RH, Heneghan MA. Liver disease in pregnancy. Lancet. 2010;375:594-605.

2. Lammert F, Marschall HU, Glantz A, Matern S. Intrahepatic cholestasis of pregnancy: molecular pathogenesis, diagnosis and management. J Hepatol. 2000;33:1012-21.

3. Geenes V, Williamson C. Intrahepatic cholestasis of pregnancy. World J Gastroenterol. 2009;15:2049-66.

4. Mullally BA, Hansen WF. Intrahepatic cholestasis of pregnancy: review of the literature. Obstet Gynecol Surv. 2002;57(1):47-52.

5. Kondrackiene J, Kupcinskas L. Intrahepatic cholestasis of pregnancy-current achievements and unsolved problems. World J Gastroenterol. 2008;14(38):5781-8.

6. Bacq Y, Sentilhes L, Reyes HB, Glantz A, Kondrackiene $\mathrm{J}$, Binder $\mathrm{T}$, et al. Efficacy of ursodeoxycholic acid in treating intrahepatic cholestasis of pregnancy: a meta-analysis. Gastroenterol. 2012;143(6):1492-501.

7. Chappell LC, Gurung V, Seed PT, Chambers J, Williamson C, Thornton JG. Ursodeoxycholic acid versus placebo, and early term delivery versus expectant management, in women with intrahepatic cholestasis of pregnancy: semifactorial randomised clinical trial. BMJ. 2012;344:e3799.

8. Pan C, Perumalswami PV. Pregnancy-related liver diseases. Clin Liver Dis. 2011;15(1):199-208.

9. Glantz A, Marschall HU, Mattsson LA. Intrahepatic cholestasis of pregnancy: Relationships between bile acid levels and fetal complication rates. Hepatol. 2004;40(2):467-74.

10. Davies MH, da Silva RC, Jones SR, Weaver JB, Elias E. Fetal mortality associated with cholestasis of pregnancy and the potential benefit of therapy with ursodeoxycholic acid. Gut. 1995;37(4):580-4.

11. Palma J, Reyes H, Ribalta J, Hernández I, Sandoval L, Almuna R, et al. Ursodeoxycholic acid in the treatment of cholestasis of pregnancy: a randomized, double-blind study controlled with placebo. J Hepatol. 1997;27(6):1022-8.

12. Rook M, Vargas J, Caughey A, Bacchetti P, Rosenthal $\mathrm{P}$, Bull L. Fetal outcomes in pregnancies complicated by intrahepatic cholestasis of pregnancy in a Northern California cohort. PLoS One. 2012;7(3):e28343.

13. Benjaminov FS, Heathcote J. Liver disease in pregnancy. Am J Gastroenterol. 2004;99:2479-88.

14. Kondrackiene J, Beuers U, Kupcinskas L: Efficacy and safety of ursodeoxycholic acid versus cholestyramine in intrahepatic cholestasis of pregnancy. Gastroenterol. 2005;129(3):894-901.

15. Glantz A, Marschall HU, Lammert F, Mattsson LÅ. Intrahepatic cholestasis of pregnancy: a randomized controlled trial comparing dexamethasone and ursodeoxycholic acid. Hepatol. 2005;42(6):1399-405.

16. Ge X, Xu YQ, Huang SH, Huang K, Mao LJ, Pan WJ, et al. Intrahepatic cholestasis of pregnancy and fetal outcomes: a prospective birth cohort study. Zhonghua Liu Xing Bing Xue Za Zhi. 2016;37(2):187-91.

17. Türkmen GG, Timur H, Yilmaz Z, Kirbas A, Daglar K, Tokmak A, et al. Effect of intrahepatic cholestasis of pregnancy on maternal serum screening tests. J Neonatal Perinatal Med. 2016;9(4):411-5.

18. Garcia-Flores J, Cañamares M, Cruceyra M, Garicano A, Espada M, Lopez A, et al. Clinical value of maternal bile Acid quantification in intrahepatic cholestasis of pregnancy as an adverse perinatal outcome predictor. Gynecol Obstet Invest. 2015;79(4):222-8.

19. Brouwers L, Koster MPH, Page-Christiaens GCML, Kemperman H, Boon J, Evers IM, et al. Intrahepatic cholestasis of pregnancy: maternal and fetal outcomes associated with elevated bile acid levels. Am J Obstet Gynecol 2015;212:100.e1-7.

20. Wikström Shemer E, Marschall HU, Ludvigsson JF, Stephansson O. Intrahepatic cholestasis of pregnancy and associated adverse pregnancy and fetal outcomes: a 12-year population-based cohort study. BJOG. 2013;120:717-23.

21. Sharma N, Panda S, Santa Singh A. Obstetric outcome during an era of active management for obstetrics cholestasis. J Obstet Gynecol India. 2016;66(1):38-41.

Cite this article as: Sharma P, Sarkar B, Majhi B. Fetal and neonatal outcomes in intrahepatic cholestasis of pregnancy. Int J Reprod Contracept Obstet Gynecol 2018;7:4056-60. 